\title{
IMPACT OF VERTICAL LEVEL DISTRIBUTIONS ON SIMULATED STRATOSPHERIC CLIMATE
}

\author{
LIU, X. Y. ${ }^{*}-$ LU, C. C. ${ }^{2}$ \\ ${ }^{1}$ College of Oceanography, Hohai University, Nanjing 210098, China \\ ${ }^{2}$ College of Meteorology and Oceanography, National University of Defense Technology \\ Nanjing 211101, China \\ ${ }^{*}$ Corresponding author \\ e-mail:xyliu@hhu.edu.cn \\ (Received $17^{\text {th }}$ May 2019; accepted $16^{\text {th }}$ Jul 2019)
}

\begin{abstract}
The climate of stratosphere was simulated with a spectral element model under two different vertical level distributions, one had 28 levels with $0.3 \mathrm{hPa}$ at the top of the atmosphere and another 66 levels with $4.5 \times 10^{-6} \mathrm{hPa}$ at the top boundary. Simulation results were validated against ERA-Interim reanalysis dataset from ECMWF (European Center for Medium-Range Weather Forecasts) and the influence of vertical level distributions on the numerical simulation of stratospheric temporal mean state had been analyzed. It is shown that the lift of upper boundary can reduce the temperature error above the height of $100 \mathrm{hPa}$ level significantly. It can also improve the simulation of seasonal variation of zonal mean zonal wind on $50 \mathrm{hPa}$ in the northern high latitudes. In both configurations, cold biases of 10-year averaged zonal mean of temperature are mainly discerned at levels around $75 \mathrm{hPa}$ at low latitudes and levels around $175 \mathrm{hPa}$ at high southern latitudes. The simulated seasonal variation of westerly at $50 \mathrm{hPa}$ in the northern hemisphere with 66-layer model coincides with results of ERA-Interim better.

Keywords: spectral element, atmospheric model, vertical resolution, numerical modeling, middle atmosphere
\end{abstract}

\section{Introduction}

With the advancement of observation technique, there have been more observations available in the stratosphere and their reliability has improved greatly as well. People have come to possess more knowledge of variation tendency of the stratospheric climate and have deepened their understanding on the anthropogenic and natural factors that contribute to the climate change. People also have a more comprehensive understanding of the connections in processes of dynamics and radiation thermodynamics between the changes in the stratosphere and that specific to the earth's surface. For example, it has been realized that temperature change in the stratosphere is an important component of the global climate change, and its trend can give evidence for the separation of natural and anthropogenic effects contributing to the climate change (Randel et al., 2009); the vapor in the stratosphere plays an important role in the radiation balance of troposphere (Held and Soden, 2000); the stratosphere can influence the climate of the earth surface as well as its variability through the remote response mechanism between the stratosphere and the troposphere (Baldwin et al., 2007; Mitchell et al., 2013); and so on. The researches on the stratospheric climate are getting more people's attention. Of which, numerical modeling is an important direction.

In atmospheric climate models, two discretization methods, which are finite difference and spectral approximation, are mostly used to solve the partial differential equations numerically. Other methods, such as finite element, finite volume and so on are also used in some models. The advantage of finite difference is that it is simple and 
intuitive and easy for implementation of parallel computing. But it has the weakness of low accuracy compared to spectral approximation. The advantage of spectral method is that it has high accuracy. But since spectral approximation is carried out on the whole domain of computation, its local errors can disseminate to large area and it is hard to implement parallel computing basing on the idea of region splitting. The finite element method has the merits that it is suitable for seeking solution for domain of complex geometry; it is flexible and also easy for parallel computing. But it cannot give high accuracy solution as the spectral method does. The finite volume method can be used to construct discreet scheme more flexibly than the finite difference method and it share similar merits and weakness with the finite difference method. The spectral element method combines the merits of high accuracy from spectral approximation and flexibility from finite element discretization (Patera, 1984). The basic idea of performing computation with spectral element method is dividing the domain into finite elements and then solving the problem with spectral approximation within each of them. The spectral element method has been used widely in numerical modeling of geophysical fluid dynamics (Founier et al., 2004; Giraldo, 2005; Liu, 2011).

Many climate models used mainly for troposhere study cannot depict the stratosphere well due to their low height of top boundaries. In theses low top models, although reasonable mean climate can be reproduced through introducing damping at their top boundaries, which usually situate at the stratosphere, the simulated variability is small. There have been increasing evidences showing that the variability in the stratosphere has significant influence on the modes of climate variability in the troposphere and the earth surface (Norton, 2003; Charlton et al., 2004; Scaife et al., 2005). Besides, the influence of climate change on the stratospheric circulation is a hot topic in current scientific research, whereas the reactions of stratospheric circulation variation to ozone and earth surface climate are unclear to a large extent (Butchart and Scaife, 2001; Butchart et al., 2006; Abalos et al., 2015; Linz et al., 2017).

Since the paper of Boville (1984), many works on the influence of the middle atmosphere on the properties of the tropospheric simulation have been performed (for example, Boville and Cheng, 1988; Boville and Baumhefner, 1990; Sassi, 2010; Shaw and Perlwitz, 2010). Ruosteenoja (2006) studied the sensitivity of the simulations of tropospheric stationary waves to the height of the model top with a linear model. It is of necessity to study the influence of the height of model top on the simulation of stratosphere as well. In the paper, a model, in which the prognostic variables are discretized with spectral element method in the horizontal and the whole stratosphere is included, will be used to simulate the stratospheric climate under configuration of two different top boundary extensions; and, if a lid in the mesosphere versus one in the lower thermosphere makes any difference to the representation of climate in the stratosphere will be studied. The work will enrich the understanding on the sensitivity of the simulations of stratospheric climate to the height of the model top and contribute to the choice of model resolution for study of stratosphere.

\section{Materials and Methods}

The atmospheric general circulation model SEMANS (Spectral Element Model with Atmospheric Near Space resolved) (Liu et al., 2015) is used in the work. It uses a horizontal coordinate on the projection plane of cubed sphere (Ronchi et al., 1996) and a $\mathrm{p}-\sigma$ mixed vertical coordinate (Simmons and Burridge, 1981). The model prognostic 
variables are discretized with spectral element method in the horizontal and finite difference in the vertical respectively. When spectral element discretization is used, a triangular or quadrilateral basic element (Giraldo, 2005; Liu, 2011) is often adopted. SEMANS adopts a quadrilateral basic element. By using of coordinate on the projection plane of cubed sphere, SEMANS can overcome the difficulty of polar problem which is unavoidable in longitude-latitude grids. Through using of $p-\sigma$ mixed vertical coordinate, the model can treat topography easily and reduces the error from calculation of pressure gradient at high altitude of the atmosphere as well. Schemes used for physical processes are shown in Table 1.

Table 1. Schemes used for physical processes

\begin{tabular}{|c|c|}
\hline Physical process & Scheme used \\
\hline Deep convection & Zhang and McFarlane (1995) \\
\hline Shallow convection & Park and Bretherton (2009) \\
\hline Boundary layer parameterization & Nonlocal diffusion scheme (Holtslag and Boville, 1993) \\
\hline Near surface constant flux over land & Monin-Obukhov similarity theory \\
\hline $\begin{array}{l}\text { Near surface constant flux over ocean water and sea } \\
\text { ice }\end{array}$ & Bulk formula \\
\hline Thermal radiation & Ramanathan et al.(1986) \\
\hline Solar radiation for height below $60 \mathrm{~km}$ & $\delta$-Eddington approximation (Briegleb, 1992) \\
\hline Solar radiation for height between $60 \mathrm{~km}$ and $100 \mathrm{~km}$ & $\begin{array}{c}\text { Radiation heating rates from TIME-GCM (Thermosphere } \\
\text { Ionosphere Mesosphere Electrodynamics General Circulation } \\
\text { Model) (Marsh and Roble, 2002) } \\
\text { Weighted average of TIME-GCM results and output of } \\
\text { radiation transfer with } \delta \text {-Eddington approximation }\end{array}$ \\
\hline
\end{tabular}

The low boundary value, initial value and the data for validation of simulation results are all from the ERA-Interim reanalysis datasets (Simmons et al., 2007). In model validation, the reanalysis data from 1997 to 2006 will be used.

Two numerical experiments were designed. In the first one (denoted by EXP1), the model atmosphere was divided into 28 layers and the air pressure of model top is 0.3 hPa (see Fig. 1a); in the second one (denoted by EXP2), the model atmosphere was divided into 66 layers and the air pressure of model top is $4.5 \times 10^{-6} \mathrm{hPa}$ (Fig. 1b). In both numerical experiments, every projected plane was divided into 81 basic elements (Fig. 1c) and the $8^{\text {th }}$ degree Gauss-Legendre-Lobatto interpolation polynomials were used to approximate the solution with spectral method within each basic element (Fig. 1d). Since the model atmosphere in EXP2 had extended beyond $100 \mathrm{~km}$ in altitude, the radiation transfer scheme in EXP1 was no longer valid for high level atmosphere. In EXP2, radiation heating rates from TIME-GCM (Thermosphere Ionosphere Mesosphere Electrodynamics General Circulation Model) (Marsh and Roble, 2002) were used in model layers above a certain height (approximately $100 \mathrm{~km}$ ); in model layers below a certain height (approximately $65 \mathrm{~km}$ ) radiation transfer scheme identical to that of EXP1 were used; in model layers between the two heights, weighted average of TIME-GCM results and radiation transfer results of EXP2 were used (chemical processes were not considered here). The atmospheric state at 0000 UTC 1 January 1997 from the ERA-Interim datasets was used as initial values. For model layers above the height of $1 \mathrm{hPa}$ level, initial values of model variables are identical to those of the highest model layer just below the height of $1 \mathrm{hPa}$ level. The low boundary 
conditions (ground surface temperature, sea ice concentration, soil moisture etc) are all multi-year (1997-2006) means with monthly variations included. In each numerical experiment, the model was integrated for 20 years and monthly mean results were saved. If not noted specifically, the results for model output and reanalysis all denote 10 year mean case.

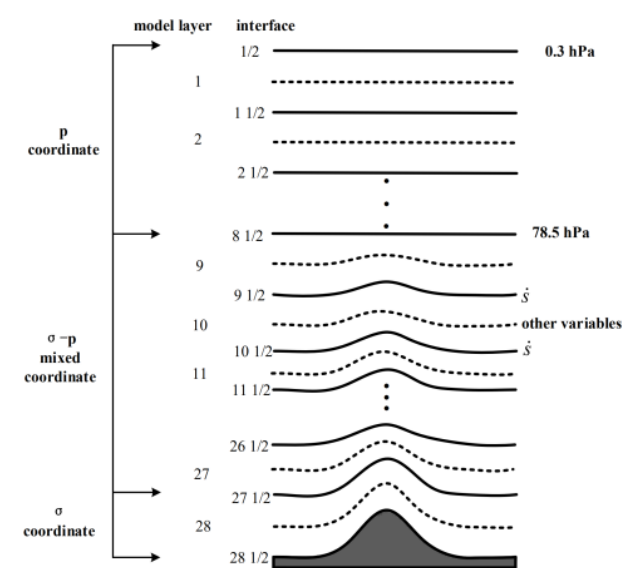

(a)

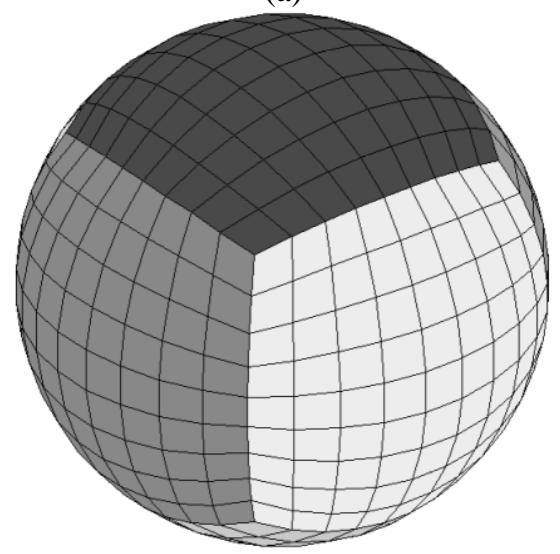

(c)

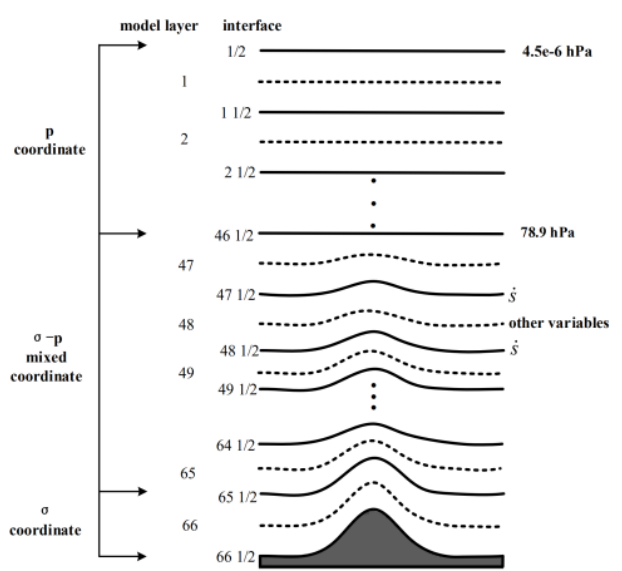

(b)

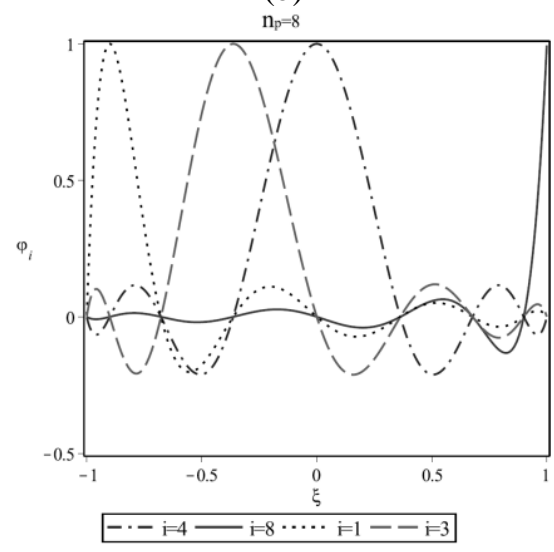

(d)

Figure 1. Discretization of SEMANS. (a) vertical division of model atmosphere in version of 28 layers, (b) vertical division of model atmosphere in version of 66 layers, (c) projected grids on cubed sphere (81 elements in each face), (d) Gauss-Legendre-Lobatto interpolation polynomials in local coordinate from -1 to 1 (with a degree of 8 )

\section{Results}

The two numerical experiments all reproduce such features as the 2-wave pattern in the northern hemisphere and the 1-wave pattern in the southern hemisphere of geopotential at $20 \mathrm{hPa}$. The strength of simulated perturbation in the northern hemisphere is weaker than that of the reanalysis data and the strength of simulated perturbation in the northern hemisphere is close to that of the reanalysis data (see Fig. 2). The patterns of geopotential reproduced by both the 28 layer version and the 66 layer version of SEMANS are similar, whereas the values from the 28 layer version are bigger (compare Fig. $2 a$ with $2 b$ ). Compared to results from reanalysis dataset, the extent encircled by the 26400 geopotential meter contour is smaller (compare Fig. $2 a$ with $2 c$ ). 


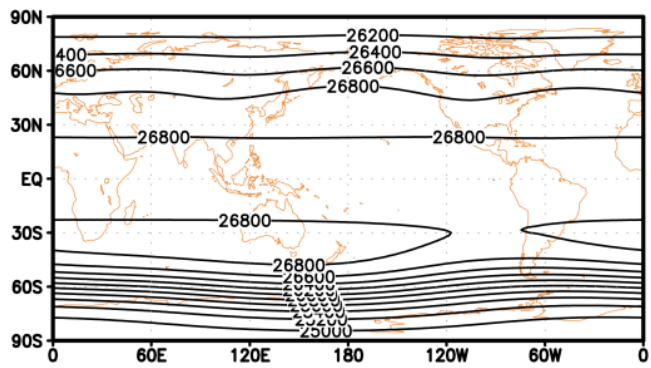

(a)

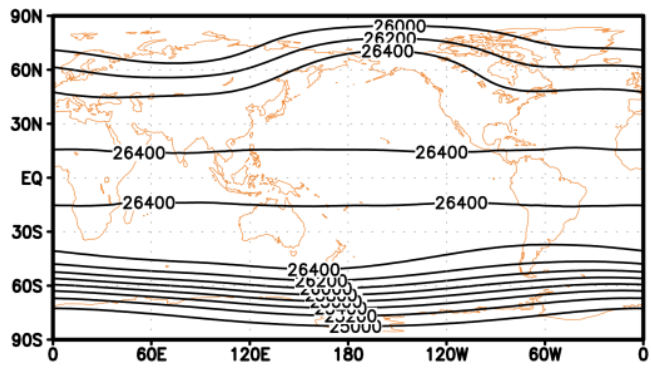

(c)

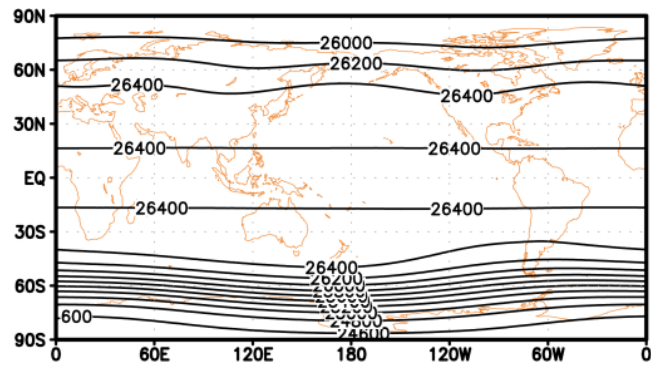

(b)

Figure 2. Distribution of geopotential height at 20hPa (unit:gpm). (a) EXP1, (b) EXP2, (c) ERA-Interim. The horrizontal axis is longitude $\left({ }^{\circ}\right)$ and the vertical axis is latitude $\left({ }^{\circ}\right)$ in each panel

From the 10-year averaged zonal mean temperature difference (Fig. 3), it can be seen that, the simulated climate features of temperature are similar from the two different configurations of SEMANS; the cold biases of zonal mean temperature in the low latitudes are mainly confined to the atmospheric levels around $75 \mathrm{hPa}$; there are cold biases above the height of $20 \mathrm{hPa}$ level in the two polar regions and the biases from the 66-layer configuration are relatively smaller; there are also severe cold biases around the $175 \mathrm{hPa}$ level in the southern high latitudes and the region near $45^{\circ} \mathrm{N}$ (compare Fig. $3 a$ with $3 b$ ).

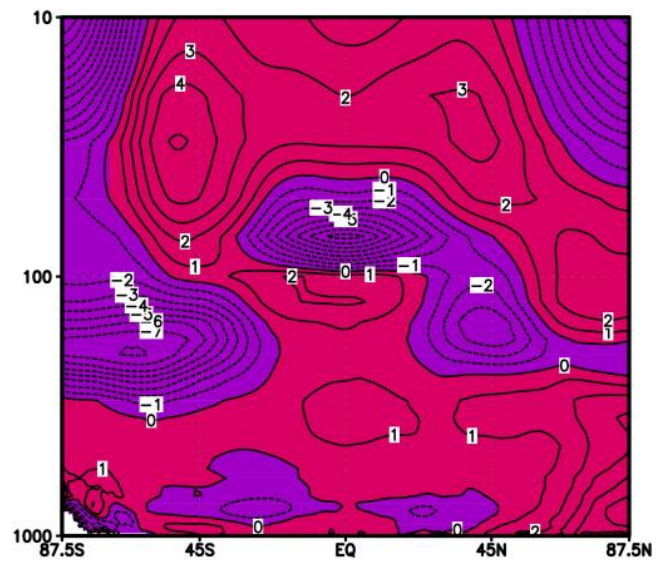

(a)

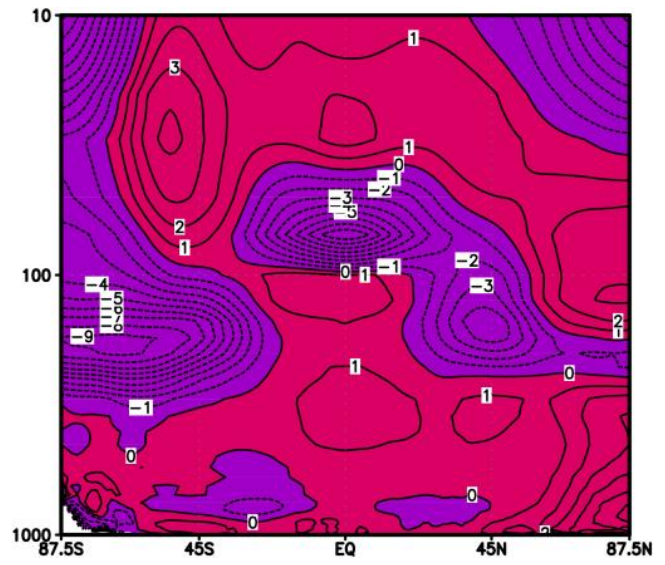

(b)

Figure 3. Difference of zonal mean temperature (unit:K) between model results and ERAInterim. (a) EXP1 minus ERA-Interim, (b) EXP2 minus ERA-Interim. The horrizontal axis is latitude $\left(^{\circ}\right)$ and the vertical axis is pressure $(\mathrm{hPa})$ in each panel 
The divisions of model atmosphere are identical within the troposphere in the two configurations. But due to the influence of differences in the height of model top and divisions of model atmosphere at higher levels, there are also differences in the reproduced temperature of troposphere, which are quite smaller compared to that of stratosphere.

From the differences of regionally averaged temperature between model results and ERA-Interim (Fig. 4), it is clear that, the simulated temperature biases varies with height in the same manner for results in EXP1 and its counterpart in EXP2; the globally averaged annual mean temperature from the 66-layer model is colder than that from the 28-layer model (see Fig. 4a); in the winter, the simulated temperatures above the height of $100 \mathrm{hPa}$ level in the high latitudes of both hemisphere are warmer in EXP2 (see Fig. $4 b$ and $4 d$ ); in the summer, the simulated temperatures above the height of $100 \mathrm{hPa}$ level in the high latitudes of both hemisphere are colder in EXP2 (see Fig. 4c and 4e). It's apparent that the biases of simulated temperature above the height of $100 \mathrm{hPa}$ level are smaller in EXP2. In contrast, the biases of simulated regionally averaged temperature in the high latitudes in both the spring and the autumn are smaller (figures not shown).

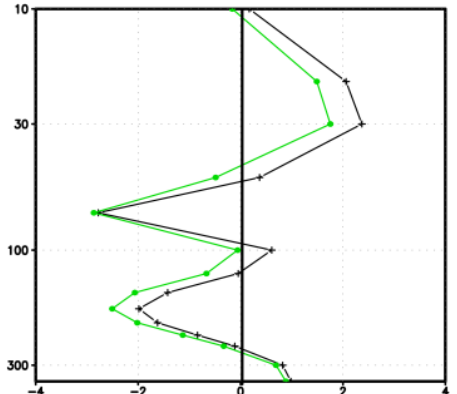

(a)

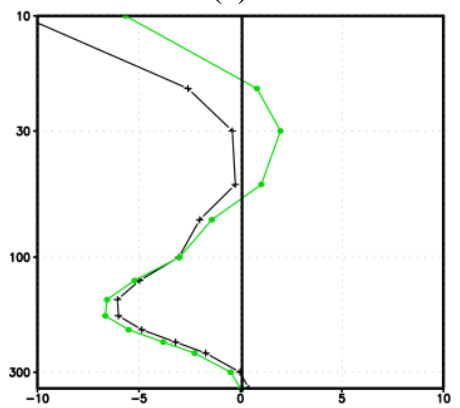

(d)

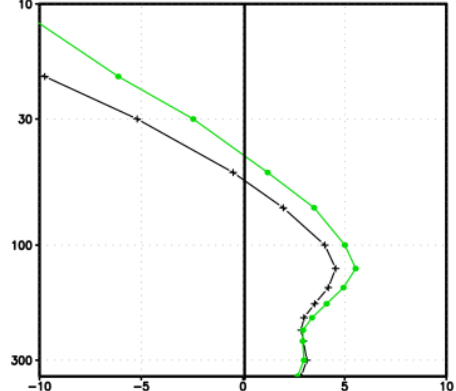

(b)

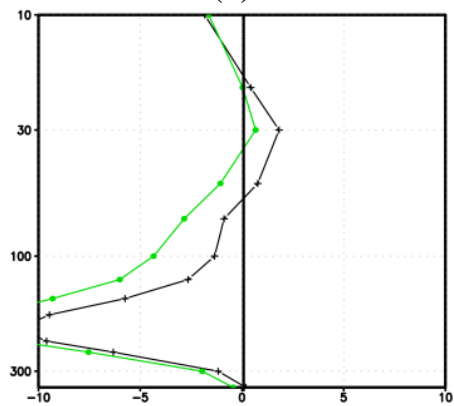

(e)

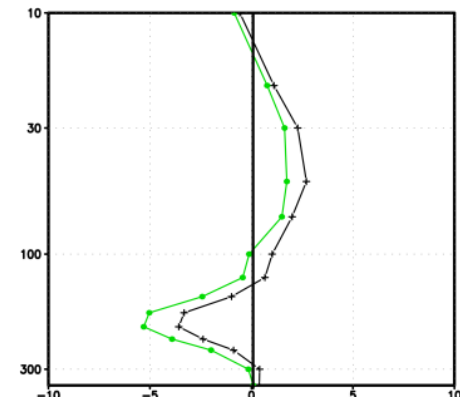

(c)

Figure 4. Differences of regionally averaged temperature between model results and ERAInterim. Cross and solid dot denote results from EXP1 minus ERA-Interim and EXP2 minus ERA-Interim respectively. (a) annual global average, (b) winter (December, January and February) $60-90^{\circ} \mathrm{N}$ average, (c) summer (June-August) $60-90^{\circ} \mathrm{N}$ average, (d) winter (JuneAugust) $60-90^{\circ} \mathrm{S}$ average, (e) summer (December, January and February) $60-90^{\circ} S$ average. The horrizontal axis is temperature difference $(K)$ and the vertical axis is pressure $(\mathrm{hPa})$ in each panel

In both numerical experiments, the major features of zonal mean zonal wind distribution (such as position of westerly belts, easterly belts and centers of strong wind) at $50 \mathrm{hPa}$ are consistent (see Fig. $5 a$ and $5 b$ ). Compared to that of ERA-Interim, the 
simulated seasonal variation of westerly in the northern hemisphere and intensity of westerly wind in the southern hemisphere by the 66-layer model agrees with that of ERA-Interim better (compare Fig. $5 a$ with $5 b$ and $5 c$ ).

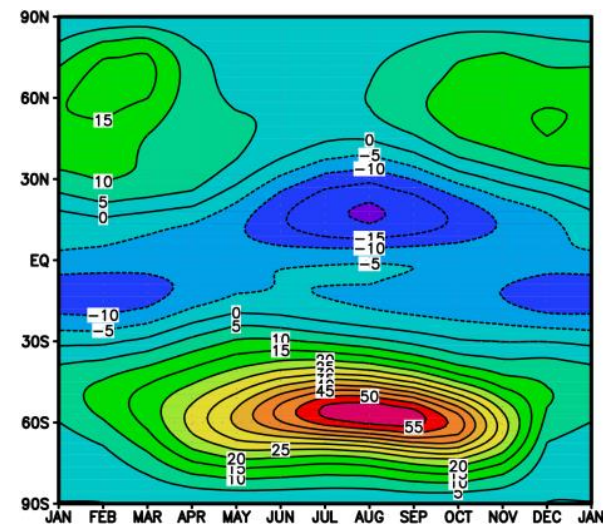

(a)

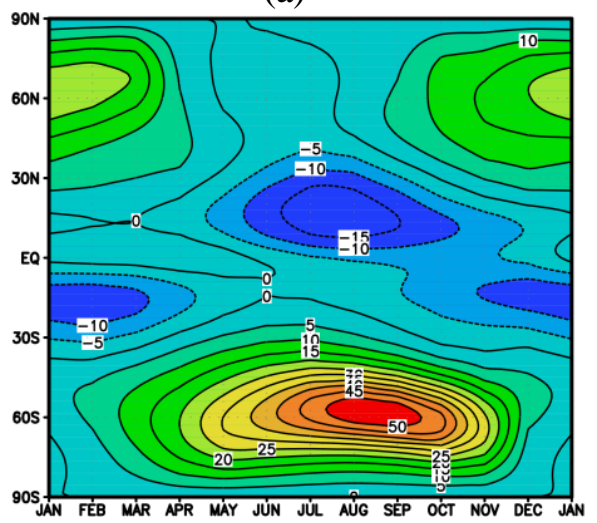

(c)

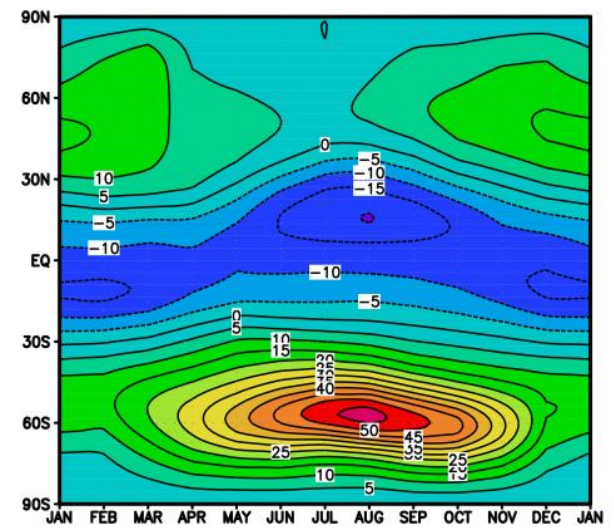

(b)

Figure 5. Distribution of zonal mean zonal wind at 50hPa (unit: $\mathrm{m} / \mathrm{s})$. (a) EXP1, (b) EXP2, (c) ERA-Interim. The horrizontal axis is time (month) and the vertical axis is latitude $\left(^{\circ}\right)$ in each panel

\section{Discussion}

In the study, a numerical atmospheric model with two configurations had been used to study the influence of vertical divisions of the model atmosphere on the simulation of stratospheric climate. All the employed schemes of physical processes except solar radiation are identical in the two configurations. There have been work on the effects of upper layer of atmosphere on simulations of stratosphere or troposphere based on results from different models (for example, Sassi et al., 2010). It would be difficult to deduce the reason of specific feature in simulated stratospheric climate from multiple models since there are many factors involved. In contrast, attribution is simple if simulations are from the same model. But work to study the influence of upper atmosphere on the simulation of stratospheric climate under the same dynamical framework are still less.

The analysis in the work was only on the mean climate. This is very limited. Any downward influence of the middle atmosphere on the troposphere is likely to happen during dynamically disturbed times. Thus, the time mean is not a sufficient measure to describe climate influence. More work of comparative analysis, such as the behavior of 
the model during sudden stratospheric warming, the features of the penetration of stratospheric anomalies in the two models, and so on, should be done in future research.

No chemical process has been included in SEMANS and the radiation process scheme is rather simplified for atmosphere of high altitude (above $65 \mathrm{~km}$ ). If the chemical processes and more complex radiation process scheme are considered, the simulation results may show new features. So, much further work needs to be done in the future research.

\section{Conclusion}

A numerical model named SEMANS had been used to study if a lid in the mesosphere versus one in the lower thermosphere makes any difference to the representation of climate in the stratosphere. It's shown that, there are apparent similarities in the simulation results from the two configurations; the cold biases of zonal mean temperature in the low latitudes are mainly confined to the atmospheric levels around $75 \mathrm{hPa}$; the globally averaged annual mean temperature from the 66-layer model is colder than that from the 28-layer model; the biases of simulated temperature above the height of $100 \mathrm{hPa}$ level are smaller in results from 66-layer version model; the simulated seasonal variation of westerly in the northern hemisphere and intensity of westerly wind in the southern hemisphere by the 66-layer model agrees with that of ERA-Interim better.

Acknowledgements. The work is sponsored by "the Fundamental Research Funds for the Central Universities" in China (Grant No. 2019B00214). The ERA-Interim reanalysis datasets were downloaded from the ECMWF data server website.

\section{REFERENCES}

[1] Abalos, M., Legras, B., Ploeger, F., Randel, W. J. (2015): Evaluating the advective Brewer-Dobson circulation in three reanalyses for the period 1979-2012. - J. Geophys. Res. 120: 7534-7554.

[2] Baldwin, M. P., Dameris, M., Shepherd, T. G. (2007): How will the stratosphere affect climate change. - Science 316: 1576-1577.

[3] Boville, B. A. (1984): The influence of the polar night jet on the tropospheric circulation in a GCM. - J. Atmos. Sci. 41: 1132-1142.

[4] Boville, B. A., Cheng, X. (1988): Upper boundary effects in a general circulation model. - J. Atmos. Sci. 45: 2591-2606.

[5] Boville, B. A., Baumhefner, D. P. (1990): Simulated forecast error and climate drift resulting from the omission of the upper stratosphere in numerical models. - Mon. Wea. Rev. 118: 1517-1530.

[6] Briegleb, B. P. (1992): Delta-Eddington approximation for solar radiation in the NCAR Community Climate Model. - J. Geophys. Res. 97: 7603-7612.

[7] Butchart, N., Scaife, A. A. (2001): Removal of chlorofluorocarbons by increased mass exchange between the stratosphere and troposphere in a changing climate. - Nature 410 : 799-802.

[8] Butchart, N., Scaife, A. A., Bourqui, M., de Grandpré, J., Hare, S. H. E., Kettleborough, J., Langematz, U., Manzini, E., Sassi, F., Shibata, K., Shindell, D., Sigmond, M. (2006): Simulations of anthropogenic change in the strength of the Brewer-Dobson circulation. Climate Dynamics 27: 727-741. 
[9] Charlton, A. J., Neill, A. O., Lahoz, W. A., Massacand, A. C. (2004): Sensitivity of tropospheric forecasts to stratospheric initial conditions. - Q. J. R. Meteorol. Soc. 130: 1771-1792.

[10] Founier, A., Taylor, M. A., Tribbia, J .J. (2004): The spectral element atmosphere model (SEAM): high-resolution parallel computation and localized resolution of regional dynamics. - Mon. Wea. Rev. 132: 726-748.

[11] Giraldo, F. X. (2005): Semi-implicit time-integrators for a scalable spectral element atmospheric model. - Q. J. R. Meteorol. Soc. 131: 2431-2454.

[12] Held, I. M., Soden, B. J. (2000): Water vapor feedback and global warming. - Annu. Rev. Energy Environ. 25: 441-475.

[13] Holtslag, A. A. M., Boville, B. A. (1993): Local versus nonlocal boundary-layer diffusion in a global climate model. - J. Climate 6: 1825-1842.

[14] Linz, M., Plumb, R. A., Gerber, E. P., Haenel, F. J., Stiller, G., Kinnison, D. E., Ming, A., Neu. J. L. (2017): The strength of the meridional overturning circulation of the stratosphere. - Nature Geoscience 10: 663-667.

[15] Liu, X. Y. (2011): Numerical simulation of typhoon movement with a regional spectral element barotropic atmospheric model. - Chinese Journal of Computational Physics 28: $35-40$.

[16] Liu, X. Y., Liu, C. Y., Yao, S. S. (2015): A spectral element model with atmospheric near space resolved (SEMANS). - Chinese Journal of Computational Physics 32: 40-50.

[17] Marsh, D., Roble, R. (2002): TIME-GCM simulations of lower-thermospheric nitric oxide seen by the halogen occultation experiment. - J. Atmos. Solar Terr. Phys. 64: 889895.

[18] Mitchell, D., Gray, L., Anstey, J., Baldwin, M., Charlton-Perez, A. (2013): The influence of stratospheric vortex displacements and splits on surface climate. - Geophys. Res. Lett. 26: $2668-2682$.

[19] Norton, W. A. (2003): Sensitivity of northern hemisphere surface climate to simulation of the stratospheric polar vortex. - Geophys. Res. Let. 30: 1627.

[20] Park, S., Bretherton, C. S. (2009): The university of washington shallow convection and moist turbulence schemes and their impact on climate simulations with the community atmosphere model. - J. Climate 22: 3449-3469.

[21] Patera, A. T. (1984): A spectral element method for fluid dynamics: laminar flow in a channel expansion. - J. Comput. Phys. 54: 468-488.

[22] Ramanathan, V., Downey, P. A. (1986): Nonisothermal emissivity and absorptivity formulation for water vapor. - J. Geophys. Res. 91: 8649-8666.

[23] Randel, W. J., Shine, K. P., Austin, J., Barnett, J., Claud, C., Gillett, N. P., Keckhut, P., Langematz, U., Lin, R., Long, C., Mears, C., Miller, A., Nash, J., Seidel, D. J., Thompson, D. W. J., Wu, F., Yoden, S. (2009): An update of observed stratospheric temperature trends. - Journal of Geophysical Research 114: D02107.

[24] Ronchi, C., Iacono, R., Paolucci, P. S. (1996): The 'cubed sphere': A new method for the solution of partial differential equations in spherical geometry. - J. Comput. Phys. 124: 93-114.

[25] Ruosteenoja, K. (2006): The impact of the height of the model top on the simulation of tropospheric stationary waves. - Q. J. R. Meteorol. Soc. 125: 677-695.

[26] Sassi, F., Garcia, R. R., Marsh, D., Hoppel, K. W. (2010): The Role of the Middle Atmosphere in Simulations of the Troposphere during Northern Hemisphere Winter: Differences between High- and Low-Top Models. - J. Atmos. Sci. 67: 3048-3064.

[27] Scaife, A. A., Knight, J. R., Vallis, G. K., Folland, C. K. (2005): A stratospheric influence on the winter NAO and North Atlantic Surface Climate. - Geophys. Res. Let. 32: L18715.

[28] Shaw, T. A., Perlwitz, J. (2010): The Impact of Stratospheric Model Configuration on Planetary-Scale Waves in Northern Hemisphere Winter. - J. Climate 23: 3369-3389. 
[29] Simmons, A. J., Burridge, D. M. (1981): An Energy and Angular-Momentum Conserving Vertical Finite-Difference Scheme and Hybrid Vertical Coordinates. - Mon. Wea. Rev. 109: 758-766.

[30] Simmons, A., Uppala, S., Dee, D., Kobayashi, S. (2007): ERA-Interim: New ECMWF reanalysis products from 1989 onwards. - ECMWF Newsletter 110: 25-35.

[31] Zhang, G. J., McFarlane, N. A. (1995): Sensitivity of climate simulations to the parameterization of cumulus convection in the Canadian Climate Centre general circulation model. - Atmosphere- Ocean 33: 407-446. 